

\section{DISCLAIMER}

This report was prepared as an account of work sponsored by an agency of the United States Government. Neither the United States Government nor any agency thereof, nor any of their employees, makes any warranty, express or implied, or assumes any legal liability or responsibility for the accuracy, completeness, or usefulness of any information, apparatus, product, or process disclosed, or represents that its use would not infringe privately owned rights. Reference herein to any specific commercial product, process, or service by trade name, trademark, manufacturer, or otherwise does not necessarily constitute or imply its endorsement, recommendation, or favoring by the United States Government or any agency thereof. The views and opinions of authors expressed herein do not necessarily state or reflect those of the United States Government or any agency thereof. 


$$
\text { CONF }-850507--7
$$

\title{
PAIRING CORRECTION OF PARTICLE-HOLE STATE DENSITIES FOR TWO KINDS OF FERMIONS*
}

\author{
C. Y. FU
}

Oak Ridge National Laboratory, Oak Ridge, Tennessee, 37831 USA

\begin{abstract}
Pairing corrections ín particle-hole (exciton) state-density formulas used in precompound nuclear reaction theories are, strictly speaking, dependent on the nuclear excitation energy $U$ and the exciton number $n$. A general formula for $(U, n)$-dependent pairing corrections has been derived in an earlier paper for exciton state-density formulas for one kind of Fermion. In the present paper, a similar derivation is made for two kinds of Fermions. It is shown that the constant-pairing-energy correction used in standard leveldensity formulas, such as $U_{0}$ in Gilbert and Cameron, is a limiting case of the present general $(U, n)$-dependent results.
\end{abstract}

\section{INTRODUCTION}

If the two-Fermion state-density formula ${ }^{1}$

$$
\omega_{2}(U)=\frac{\sqrt{\pi}}{12} \frac{\exp \left[2\left(a U^{*}\right)^{1 / 2}\right]}{a^{1 / 4} U^{* / 4}}
$$

is used in a compound-reaction cross-section calculation, what particle-hole statedensity formula should one use for a consistent precompound-reaction cross-section analysis?

In Eq. (1), the subscript 2 indicates the two-Fermion formulation in which neutrons and protons occupy cifferent sets of single particle states; the parameter $a$ is related to the single-particle state density $g$ by $a=\pi^{2} g / 6$; and the effective excitation $U^{*}$ is given by $U^{*}=U-U_{0}$, where $U$ is the excitation energy and $U_{0}$ is a constant-pairing-energy correction, such as tabulated by Gilbert and Cameron. ${ }^{1}$

*Research sponsored by the U.S. Department of Energy under contract DE-AC05-840R21400 with Martin Marietta Energy Systems, Inc. 


\section{Y.FU}

Equation (1) was derived under the assumption that $g_{\pi}=g_{y}=g / 2$ and $g_{\pi}, g_{m}$ and $g$ are constants. The quantities $g_{\pi}$ and $g_{r}$ are, respectively, singleproton and single-neutron state densities. Making the same assumption and introducing the pairing correction $P_{2}$ into the two-Fermion exciton state-density formula of Williams, ${ }^{2}$ we have

$$
\omega_{2}\left(p_{\pi}, h_{\pi}, p_{\eta}, h_{n} U\right)=\left(\frac{g}{2}\right)^{n} \quad \frac{\left(U-B-P_{2}\right)^{n-1}}{p_{\pi} ! h_{\pi} ! p_{n} ! h_{n} !(n-1) !},
$$

where $n$ is the exciton number equaling $p_{\pi}+h_{z}+p_{z}+h_{m} p_{\pi}$ the proton-particle number, $h_{\pi}$ the proton-hole number, $p_{z}$ the neutron-particle number, and $h_{y}$ the neutron-hole number. The quantity $B$ is the correction factor for the Pauli exclusion principle. ${ }^{2}$ If $U_{0}=0$ and $P_{2}=0$, Eqs. (1) and (2) are based on the same assuruptions and are consistent in principle. In fact, it can be shown numerically that if $U_{0}=0$ and $P_{2}=0$,

$$
\omega_{2}(U)-\sum_{\substack{p_{\pi}=h_{\pi} \\ p_{v}=h_{\nu} \ldots}} \omega_{2}\left(p_{\pi}, h_{\pi}, p_{p}, h_{v}, U\right)
$$

within $10 \%$. This small difference is probably due to approximations in deriving the Pauli correction in Eq. (2).

We now face the questions of (1) what the value of $P_{2}$ is and (2) whether the two pairing correction factors $U_{0}$ and $P_{2}$ can be related in a consistent manner. The goal of this paper is to answer these two questions.

\section{PAIRING CORRECTIONS IN THE ONE-FERMION FORMULAS}

The pairing correcinn $D_{1}(U, n)$ for the exciton state-density formula in the oneFermion formulation sis rived earlier ${ }^{3}$ can be utilized for deriving $P_{2}$ and is summarized below.

The total state density in the one-Fermion formulation is given by

$$
\omega_{1}(U)=\frac{\exp \left[2\left(a U^{*}\right)^{1 / 2}\right]}{\sqrt{48} U^{*}},
$$

where $U^{*}$ is $U-U_{0}$. The corresponding exciton state density is given by

$$
\omega_{1}(p, h, U)=\frac{g^{n}\left(U-A^{0}-P_{1}\right)^{n-1}}{p ! h !(n-1) !},
$$


where $p$ is the particle number, $h$ is the hole number, and $n$ is the exciton number equaling $p+h$. The quantity $A^{\prime}$ is a modified correction factor for the Pauli exclusion principle. ${ }^{3}$ The pairing correction $P_{1}(U, n)$ is given by

$$
P_{1}(U, n)=\frac{g}{4}\left[\Delta_{0}^{2}-\Delta^{2}(U, n)\right],
$$

where $\Delta_{0}$ is the ground-state pairing gap. In Eq. (6) the quantity $\Delta(U, n)$ is the excited-state pairing gap calculated from the pairing theory ${ }^{4}$ using $\Delta_{0}$ and $g$ as input parameters. The resulting values of $\Delta(U, n)$ were parameterized as simple functions of $U, n, \Delta_{0}$ and $g$. Depending upon the values of $U$ and $n$, the pairing correction $P_{1}(U, n)$ has values lying tetween 0 and $U_{0}$.

It has been shown $\mathrm{n}^{3}$ that the pairing correction $U_{0}$ for the total state density is equal to the value of $P_{1}(U, n)$ evaluated along the most probable exciton number, $\hat{n}$ for $U>3.15 U_{0}$. That is to say,

$$
U_{0}=P_{1}(U, \hat{n})=\frac{1}{4} g \Delta_{0}^{2}
$$

This relation provides a means of calculating $\Delta_{0}$ from a given $U_{0}$, which is well understood. It has further been shown that even for $U<3.15 U_{0}$, Eq. (7) is still sufficiently accurate. Therefore, the pairing correction $P_{1}(U, n)$ does not require any additional parameter and is consistent with $U_{0}$.

\section{PAIRING CORRECTION IN THE TWO-FERMION FORMULAS}

The major steps in the present development and the main results are summarized in this section.

1. It is assumed that there is no pairing interaction between the proton $(\pi)$ and the neutron ( $\nu$ ) systems, so that the pairing correction $\boldsymbol{P}_{2}$ can be written as a sum of independent contributions from two one-Fermion systems:

$$
P_{2}\left(U, n_{\pi}, n_{\nu}\right)=P_{1}\left(U_{*}, n_{\pi}\right)+P_{1}\left(U_{\nu}, n_{\nu}\right) .
$$

2. The seven pairing equations contain three for the protons and three for the neutrons. Each set of the three equations is identical to that of the oneFermion model. ${ }^{2}$ The six equations are coupled by the same temperature and by the seventh equation: $U=U_{x}+U_{r}$. The seven equations are to be solved simultaneously with the seven input quantities: $g_{\pi}, g_{m}, \Delta_{0 \pi}, \Delta_{0}, n_{\pi}, n_{n}$ and $U$. The solutions of interest to the present problem are $U_{m}, U_{m} \Delta_{r}\left(U_{\tau}, n_{\tau}\right)$, and $\Delta_{v}\left(U_{m} n_{v}\right)$, which are used in Eq. (8) for calculating $P_{2}$. 


\section{Y. FU}

3. It is shown that under the approximation $\Delta_{0 \pi}=\Delta_{0 y}$ (the mean-gap approximation), the seven pairing equations can be solved approximately once for all nuclides (all combinations of $g$ and $U_{0}$, see below) with an accuracy of a few percent for $P_{2}$. The energies $U_{\pi}$ and $U_{\text {, }}$ are first determined by simple methods (essentially proportional to $n_{\pi}$ and $n_{p}$ ) satisfying the isothermal requirement; then the results given above for the one-fermion model can be utilized.

4. The constant-pairing-energy correction $U_{0}$ for the total state density can be equated to $P_{2}$ evaluated along the most probable exciton number, $\hat{n}=\hat{n}_{\pi}+\hat{n}_{r}$. This is to say that the exciton state at $\hat{n}$ is the most dominant component of the total.(compound) state. We then have

$$
U_{0}=P_{2}\left(U, \hat{n}_{r}, \hat{n}_{\nu}\right)
$$

5. From Eqs. (6), (8), and (9), we obtain

$$
\Delta_{0 \pi}^{2}=\Delta_{0 y}^{2}=4 U_{0} / g
$$

Therefore, with the mean-gap approximation, the total state density, Eq. (1), the $p$ - $h$ state density, Eq. (2), and the generalized pairing correction $P_{2}\left(U, n_{\pi}, n_{\nu}\right)$ are all defined consistently by the same two parameters $g$ and $U_{0}$.

\section{CONCLUSION}

It has been shown that the total state-density formula, Eq. (1), and the particle-hole state-density formula, Eq. (2), can be calculated consistently by the same two parameters, namely, the single-particle state density $g$ and the constant-pairi $z$ energy correction $U_{0}$. Although the pairing correction $P_{2}$ in the particle-hole statedensity formula depends on the excitation energy $U$ and the exciton numbers $n_{\pi}$ and $n_{n}$ the value of $P_{2}$ can be calculated from $g$ and $U_{0}$ as well. Therefore, if Eq. (1) is used in a compound-reaction cross-section calculation, Eq. (2) can be used consistently in a simultaneous precompound-reaction analysis.

\section{REFERENCES}

1. A. Gilbert and A. W. G. Cameron, Can. J. Phys., 43, 1446 (1965).

2. F. C. Williams, Jr., Nucl. Phys., A166, 231 (1971).

3. C. Y. Fu, Nucl. Sci. Eng., 86, 344 (1984).

4. L. G. Moretto, Nucl. Phys., A243, 77 (1975). 